\title{
HEAT CONDUCTION PROBLEM IN A TWO-LAYERED HOLLOW CYLINDER BY USING THE GREEN'S FUNCTION METHOD
}

\author{
Stanisław Kukla, Urszula Siedlecka \\ Institute of Mathematics, Czestochowa University of Technology \\ Czestochowa, Poland \\ stanislaw.kukla@im.pcz.pl,urszula.siedlecka@im.pcz.pl
}

\begin{abstract}
In this paper, a solution of the heat conduction problem in a two-layered hollow cylinder by using the Green's function method is presented. The considerations concern the heat conduction in the radial direction while taking into account time-dependent boundary conditions. The continuity condition of the temperature at the dividing surface of the layers is written in the form of a Volterra integral equation.
\end{abstract}

Keywords: heat conduction problem, two-layered cylinder, Green's function method

\section{Introduction}

The problems of the heat conduction in hollow and solid composite cylinders, in recent years, were the subject of many studies (cf. refs [1-3]). A solution of the heat conduction problem in a composite multi-layered circular cylinder can be determined by using analytical methods. In the papers [1-2] the solution of the problem was obtained by using the Laplace transform. The inverse transform has been numerically determined. However, the calculations of the inverse transform lead often to numerical instabilities and that way the search for new methods to avoid the possible instability in numerical computation is purposeful. An analytical method for solving the heat conduction problem in a composite circular cylinder, which is an extension of an approach used in Cartesian coordinates, is proposed by $\mathrm{Lu}$ et al. in reference [3]. In the paper the temperature solution for $n$-layered finite cylinder by application of the Laplace transform has been obtained and the closed form solution as the real part of a function is given. The other approach to solve the problems is the use of the properties of Green's functions. The examples of applications of the method are given by Beck et al. in the book [4]. The temperature solutions in multi-layer bodies by using the Green's function method are presented by Haji-Sheikh and Beck in paper [5]. The study concerns the three-dimensional heat conduction with examples of a two-layer body in Cartesian coordinates.

In the present paper, the solution of the problem of heat conduction in radial direction in a two-layered circular cylinder is proposed. The solution is obtained by the use of the Green's function method. 


\section{Formulation of the problem}

We consider a hollow two-layered cylinder in which the heat transfer in a radial direction is held (Fig. 1). The radial heat conduction in the layers are governed by the differential equations:

$$
\frac{\partial^{2} T_{i}}{\partial r^{2}}+\frac{1}{r} \frac{\partial T_{i}}{\partial r}=\frac{1}{k_{i}} \frac{\partial T_{i}}{\partial t}, \quad r \in\left[r_{i-1}, r_{i}\right], \quad i=1,2
$$

where $T_{i}$ is the temperature in $i$-th layer, $r$ is the radial coordinate, $t$ is time and $k_{i}$ is the thermal diffusivity of $i$-th layer.
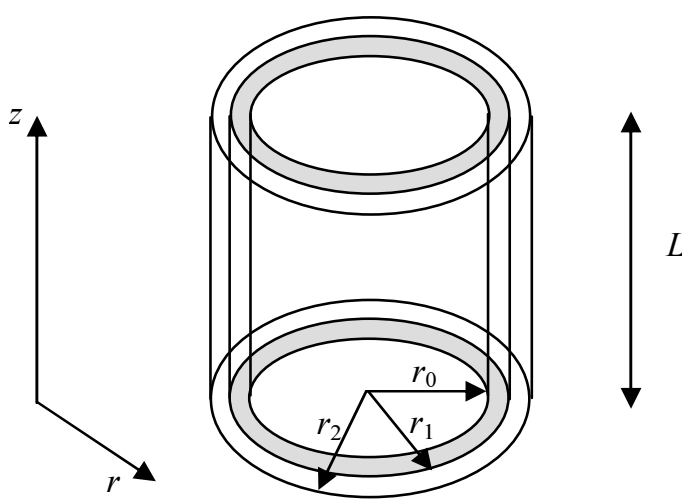

Fig. 1. The sketch of the hollow cylinder

We assume zero initial condition and boundary conditions which provide the free heat exchange at the inner $\left(r=r_{0}\right)$ and outer $\left(r=r_{2}\right)$ surfaces of the cylinder

$$
\begin{gathered}
T_{i}(0, r)=0, \quad r \in\left[r_{i-1}, r_{i}\right], \quad i=1,2 \\
-\lambda_{1} \frac{\partial T_{1}}{\partial r}\left(t, r_{0}\right)=-\alpha_{+}\left(T_{1}\left(t, r_{0}\right)-T_{+}(t)\right) \\
-\lambda_{2} \frac{\partial T_{2}}{\partial r}\left(t, r_{2}\right)=-\alpha_{\infty}\left(T_{\infty}(t)-T_{2}\left(t, r_{2}\right)\right)
\end{gathered}
$$

where $\alpha_{+}, \alpha_{\infty}$ are the surface heat transfer coefficients, $\lambda_{1}, \lambda_{2}$ are thermal conductivities and $T_{+}, T_{\infty}$ are the time-dependent boundary temperatures.

Moreover, at the contact surface of the layers, the continuity conditions are in force 


$$
\begin{gathered}
T_{1}\left(t, r_{1}\right)=T_{2}\left(t, r_{1}\right) \\
\lambda_{1} \frac{\partial T_{1}}{\partial r}\left(t, r_{1}\right)=\lambda_{2} \frac{\partial T_{2}}{\partial r}\left(t, r_{1}\right)
\end{gathered}
$$

\section{Derivation of the temperature field}

We determine the temperature $T_{1}$ and $T_{2}$ in the layers of the cylinder by using properties of Green's functions which correspond to the problem (1)-(6). The Green's functions $G_{1}$ and $G_{2}$ satisfy the auxiliary equation

$$
\frac{\partial^{2} G_{i}}{\partial r^{2}}+\frac{1}{r} \frac{\partial G_{i}}{\partial r}+\frac{1}{k_{i} r} \delta\left(r-r^{\prime}\right) \delta(t-\tau)=\frac{1}{k_{i}} \frac{\partial G_{i}}{\partial t}, \quad r \in\left[r_{i-1}, r_{i}\right], \quad i=1,2
$$

and the homogeneous boundary conditions and zero initial condition

$$
\begin{gathered}
-\lambda_{1} \frac{\partial G_{1}}{\partial r}\left(t, r_{0} ; \tau, r^{\prime}\right)=-\alpha_{+} G_{1}\left(t, r_{0} ; \tau, r^{\prime}\right) \\
\frac{\partial G_{1}}{\partial r}\left(t, r_{1} ; \tau, r^{\prime}\right)=\frac{\partial G_{2}}{\partial r}\left(t, r_{1} ; \tau, r^{\prime}\right)=0 \\
-\lambda_{2} \frac{\partial G_{2}}{\partial r}\left(t, r_{2} ; \tau, r^{\prime}\right)=\alpha_{\infty} G_{2}\left(t, r_{2} ; \tau, r^{\prime}\right) \\
G_{i}\left(0, r ; \tau, r^{\prime}\right)=0, \quad r \in\left[r_{i-1}, r_{i}\right], \quad i=1,2
\end{gathered}
$$

In equation (7) the $\delta(\cdot)$ denotes a Dirac delta function.

The derivation of the solution of the problem (7)-(11) we begin with use of the reciprocity relation [4]

$$
G_{i}\left(t, r ; \tau, r^{\prime}\right)=G_{i}\left(-\tau, r^{\prime} ;-t, r\right), \quad i=1,2
$$

Taking into account the relation (12) in equation (7), one obtains

$$
\frac{\partial^{2} G_{i}}{\partial r^{\prime 2}}+\frac{1}{r^{\prime}} \frac{\partial G_{i}}{\partial r^{\prime}}+\frac{1}{k_{i} r^{\prime}} \delta\left(r-r^{\prime}\right) \delta(t-\tau)=-\frac{1}{k_{i}} \frac{\partial G_{i}}{\partial \tau}, \quad i=1,2
$$

The temperature equations (1) we write also in terms of $r^{\prime}$ and $\tau$ as

$$
\frac{\partial^{2} T_{i}}{\partial r^{\prime 2}}+\frac{1}{r^{\prime}} \frac{\partial T_{i}}{\partial r^{\prime}}=\frac{1}{k_{i}} \frac{\partial T_{i}}{\partial \tau}, \quad i=1,2
$$


Multiplying equation (13) by $T_{i}$ and equation (14) by $G_{i}$ and subtracting the obtained equations, one gives $(i=1,2)$

$$
\frac{1}{r^{\prime}} \frac{\partial}{\partial r^{\prime}}\left(r^{\prime} \frac{\partial T_{i}}{\partial r^{\prime}}\right) G_{i}-\frac{1}{r^{\prime}} \frac{\partial}{\partial r^{\prime}}\left(r^{\prime} \frac{\partial G_{i}}{\partial r^{\prime}}\right) T_{i}-\frac{1}{k_{i} r^{\prime}} \delta\left(r-r^{\prime}\right) \delta(t-\tau) T_{i}=\frac{1}{k_{i}} \frac{\partial\left(G_{i} T_{i}\right)}{\partial \tau}
$$

Next, we integrate equation (15) with respect to $r^{\prime}$ from $r_{i-1}$ to $r_{i}(i=1,2)$ and integrate with respect to $\tau$ from 0 to $t$. The result is

$$
\begin{array}{r}
\int_{0}^{t} \int_{r_{i-1}}^{r_{i}}\left[\frac{\partial}{\partial r^{\prime}}\left(r^{\prime} \frac{\partial T_{i}}{\partial r^{\prime}}\right) G_{i}-\frac{\partial}{\partial r^{\prime}}\left(r^{\prime} \frac{\partial G_{i}}{\partial r^{\prime}}\right) T_{i}-\frac{1}{k_{i}} \delta\left(r-r^{\prime}\right) \delta(t-\tau) T_{i}\right] d r^{\prime} d \tau= \\
=\frac{1}{k_{i}} \int_{0}^{t} \int_{r_{i-1}}^{r_{i}} r^{\prime} \frac{\partial\left(G_{i} T_{i}\right)}{\partial \tau} d r^{\prime} d \tau, \quad i=1,2
\end{array}
$$

Using now the properties of the Dirac delta function, we obtain

$$
\begin{aligned}
& T_{i}(t, r)=k_{1} \int_{0}^{t} \int_{r_{i-1}}^{r_{i}}\left[\frac{\partial}{\partial r^{\prime}}\left(r^{\prime} \frac{\partial T_{i}}{\partial r^{\prime}}\right) G_{i}-\frac{\partial}{\partial r^{\prime}}\left(r^{\prime} \frac{\partial G_{i}}{\partial r^{\prime}}\right) T_{i}\right] d r^{\prime} d \tau-\left.\int_{r_{i-1}}^{r_{i}} r^{\prime}\left(G_{i} T_{i}\right)\right|_{\tau=0} ^{\tau=t} d r \\
& i=1,2
\end{aligned}
$$

Integrating by parts the integral on the right-hand side of equation (17), and next using the boundary conditions (3)-(4) and (8)-(10), we have

$$
T_{i}(t, r)=k_{i} \int_{0}^{t} r^{\prime}\left[\frac{\partial T_{i}}{\partial r^{\prime}} G_{i}-\frac{\partial G_{i}}{\partial r^{\prime}} T_{i}\right]_{r^{\prime}=r_{i-1}}^{r^{\prime}=r_{i}} d \tau, \quad i=1,2
$$

Applying the boundary conditions (3) and (4) in equation (18), gives

$$
\begin{aligned}
& T_{1}(t, r)=k_{1} \int_{0}^{t}\left[r_{1} \frac{\partial T_{1}\left(\tau, r_{1}\right)}{\partial r^{\prime}} G_{1}\left(t, r ; \tau, r_{1}\right)+r_{0} \frac{\alpha_{+}}{\lambda_{1}} T_{+}(\tau) G_{1}\left(t, r ; \tau, r_{0}\right)\right] d \tau \\
& T_{2}(t, r)=k_{2} \int_{0}^{t}\left[r_{2} \frac{\alpha_{\infty}}{\lambda_{2}} T_{\infty}(\tau) G_{2}\left(t, r ; \tau, r_{2}\right)-r_{1} \frac{\partial T_{2}\left(\tau, r_{1}\right)}{\partial r^{\prime}} G_{2}\left(t, r ; \tau, r_{1}\right)\right] d \tau
\end{aligned}
$$

The next step in the derivation of the temperature $T_{1}$ and $T_{2}$ is to determine the unknown derivatives $\frac{\partial T_{1}}{\partial r^{\prime}}$ and $\frac{\partial T_{2}}{\partial r^{\prime}}$ at $r^{\prime}=r_{1}$. In order to do this, the continuity 
conditions (5)-(6) will be used. Because from (6) we have $\frac{\partial T_{2}}{\partial r^{\prime}}=\frac{\lambda_{1}}{\lambda_{2}} \frac{\partial T_{1}}{\partial r^{\prime}}$, then using condition (5), we obtain

$$
\begin{aligned}
& k_{1} \int_{0}^{t}\left[r_{1} \frac{\partial T_{1}\left(\tau, r_{1}\right)}{\partial r^{\prime}} G_{1}\left(t, r_{1} ; \tau, r_{1}\right)+r_{0} \frac{\alpha_{+}}{\lambda_{1}} T_{+}(\tau) G_{1}\left(t, r_{1} ; \tau, r_{0}\right)\right] d \tau \\
& =k_{2} \int_{0}^{t}\left[r_{2} \frac{\alpha_{\infty}}{\lambda_{2}} T_{\infty}(\tau) G_{2}\left(t, r_{1} ; \tau, r_{2}\right)-r_{1} \frac{\lambda_{1}}{\lambda_{2}} \frac{\partial T_{1}\left(\tau, r_{1}\right)}{\partial r^{\prime}} G_{2}\left(t, r_{1} ; \tau, r_{1}\right)\right] d \tau
\end{aligned}
$$

The equation (20) can be written in the form:

$$
\int_{0}^{t} K(t, \tau) y(\tau) d \tau=F(t)
$$

where $y(\tau)=\frac{\partial T_{1}\left(\tau, r_{1}\right)}{\partial r^{\prime}}$ and $K(t, \tau)=k_{1} r_{1} G_{1}\left(t, r_{1} ; \tau, r_{1}\right)+k_{2} r_{1} \frac{\lambda_{1}}{\lambda_{2}} G_{2}\left(t, r_{1} ; \tau, r_{1}\right)$ and

$$
F(t)=\int_{0}^{t}\left[k_{2} r_{2} \frac{\alpha_{\infty}}{\lambda_{2}} T_{\infty}(\tau) G_{2}\left(t, r_{1} ; \tau, r_{2}\right)-k_{1} r_{0} \frac{\alpha_{+}}{\lambda_{1}} T_{+}(\tau) G_{1}\left(t, r_{1} ; \tau, r_{0}\right)\right] d \tau
$$

Equation (21), as the Volterra integral equation of the first kind [6], will be solved by using a quadrature method. Finally, the temperatures $T_{1}$ and $T_{2}$ in the layers of the cylinder are given by equations:

$$
\begin{aligned}
& T_{1}(t, r)=k_{1} \int_{0}^{t}\left[r_{1} y(\tau) G_{1}\left(t, r ; \tau, r_{1}\right)+r_{0} \frac{\alpha_{+}}{\lambda_{1}} T_{+}(\tau) G_{1}\left(t, r ; \tau, r_{0}\right)\right] d \tau \\
& T_{2}(t, r)=k_{2} \int_{0}^{t}\left[r_{2} \frac{\alpha_{\infty}}{\lambda_{2}} T_{\infty}(\tau) G_{2}\left(t, r ; \tau, r_{2}\right)-r_{1} \frac{\lambda_{1}}{\lambda_{2}} y(\tau) G_{2}\left(t, r ; \tau, r_{1}\right)\right] d \tau
\end{aligned}
$$

In order to compute the temperatures $T_{1}$ and $T_{2}$ we use a quadrature method to numerical calculation of integrals occurring in equations (21) and (23). After using the quadrature rule, the equation (21) gives

$$
\sum_{j=1}^{n} A_{j} K\left(t, t_{j} \cdot h\right) y\left(t_{j} \cdot h\right) d \tau=F(t)
$$


where $t_{j}$ are the points of integration and $A_{j}$ are the weights of the quadrature rule. Substituting successively: $t=t_{i} \cdot h, i=1, \ldots, n$, in equation (24) we obtain a system of equations with unknowns $y_{i}=y\left(t_{i} \cdot h\right), i=1, \ldots, n$. The equation system can be written in the matrix form

$$
\mathbf{A} \cdot \mathbf{Y}=\mathbf{F}
$$

where $\quad \mathbf{A}=\left[A_{j} K\left(t_{i}, t_{j}\right)\right]_{1 \leq i, j \leq n}, \quad \mathbf{Y}=\left[\begin{array}{llll}y_{1} & y_{2} & \ldots & y_{n}\end{array}\right]^{T}, \quad \mathbf{F}=\left[F\left(t_{1}\right) F\left(t_{2}\right) \ldots F\left(t_{n}\right)\right]^{T}$. Next, the solution of the equation (25) is used in discretized form of equations (23) for the calculation of temperatures $T_{1}, T_{2}$ in the composite cylinder.

\section{Conclusions}

The solution of the heat conduction problem in a two-layered hollow cylinder by using the properties of the corresponding Green's functions is determined. The continuity condition on the interface of the cylinder layers leads to the Volterra integral equation of the first kind which can be numerically solved. Although the problem concerns the heat conduction in radial direction, the formulation and solution of the problem can be easily expanded to the heat conduction in axis direction of the cylinder.

\section{References}

[1] Nezhad Y.R., Asemi K., Akhlaghi M., Transient solution of temperature field in functionally graded hollow cylinder with finite length using multi-layered approach, International Journal of Mechanics and Materials in Design 2011, 7, 71-82.

[2] Garbai L., Krope J., Mehes S., Bartal I., Transient heat conduction in composite systems, Proceedings of the 4th WSEAS International Conference on Heat Transfer, Thermal Engineering and Environment, Elounda, Greece, August 21-23, 2006, 372-379.

[3] Lu X., Tervola P., Viljanen M., Transient analytical solution to heat conduction in composite circular cylinder, International Journal of Heat and Mass Transfer 2006, 49, 341-348.

[4] Beck J.V., Cole K.D., Haji-Sheikh A., Litkouhi B., Heat Conduction Using Green's Functions, Hemisphere, Washington DC, 1992.

[5] Haji-Sheikh A., Beck J.V., Temperature solution in multi-dimensional multi-layer bodies, International Journal of Heat and Mass Transfer 2002, 45, 1865-1877.

[6] Grzymkowski R., Hetmaniok E., Słota D., Wykłady z modelowania matematycznego, Wydawnictwo Pracowni Komputerowej Jacka Skalmierskiego, Gliwice 2002. 